\title{
Relationship Between Sleep Quality and Overweight and Obesity in Women of Reproductive Age Referred to Health Centers in Yazd
}

\author{
Fereshte Salarinia ${ }^{1}$, Soheila Bani ${ }^{2 *}$, Mohammad Asghari Jafarabadi ${ }^{3}$, Shirin Hasanpoor ${ }^{4}$, Shamsi \\ Abasalizadeh $^{1}$, Khalil Ansari ${ }^{5}$
}

\begin{abstract}
Objectives: The present study aimed at determining the relationship between sleep quality and overweight and obesity in women of reproductive age referred to health centers and bases in Yazd in 2014-2015.

Materials and Methods: A total of 450 women of reproductive age with a body mass index (BMI) of $>18.5$ were participated in this study and divided into 3 equal groups $(\mathrm{n}=150)$ of normal weight, overweight, and obese. Instruments used in this study were the Pittsburgh Sleep Quality Index (PSQI) and a socio-demographic questionnaire. Data from the questionnaires were completed by the participants and analyzed using statistical methods.

Results: In this study, $66 \%$ of normal weight women of reproductive age had an acceptable sleep quality, while $77 \%$ of overweight and $93 \%$ of obese women of reproductive age had poor sleep quality, respectively. The results of this study showed that a significant correlation existed between BMI of normal weight women of reproductive age and the components of sleep quality $(P=0.001)$. An inverse significant relationship was also found between BMI of overweight women of reproductive age and the components of sleep quality $(P<0.05)$ and finally, an inverse significant relationship was also found between BMI of obese women of reproductive age and the components of sleep quality $(P<0.05)$. A significant relationship also existed between socio-demographic and components of sleep quality $(P<0.05)$. It seems that among the components of sleep quality, the effect of sleep disorder, impaired daily functioning, and consumption of hypnotic drugs was higher than sleep duration and good sleep in overweight and obesity.

Conclusion: The results of this study revealed that women of reproductive age with overweight and obesity have poor sleep quality. Among components of sleep quality, sleep disorder, impaired daily functioning, and consumption of hypnotic drugs have the most important role in reducing sleep quality. It seems that change in lifestyle can improve sleep quality and thus can reduce BMI.

Keywords: Obesity, Overweight, Sleep, Women
\end{abstract}

\section{Introduction}

Sleep plays a great role in maintaining health, and sleep deprivation inappropriately affects metabolic and endocrine function (1). Short sleep has become a hallmark of modern societies in adults and children in recent years. Sleep disorder and poor quality sleep are associated with chronic pulmonary hypertensive diseases which in turn are associated with lower quality of life (2). Chronic lack of sleep due to lifestyle changes is a common problem in a modern society (3). Short-term sleep disorder or sleep restriction leads to insulin resistance (1), and short sleep duration is associated with type 2 diabetes, hypertension, cardiac disease, obesity, and increased risk of overall mortality (4-6).

The prevalence of obesity is increasing in the world. It is estimated that the number of obese people in the world was around 1.5 billion in 2012 and now is rising (7). In a review article by Flegal et al, the prevalence of obesity was $35.8 \%$ in women and $35.5 \%$ in men in the United States
(8). In another review study, the prevalence of obesity was $6.2 \%-36.5 \%$ in women and $4 \%-28.3 \%$ in men in Europe (9). In a study by Moghimi-Dehkordi et al in Tehran, the prevalence of overweight and obesity was $38.3 \%$ and $17.5 \%$, respectively $(10)$.

Obesity has many social and medical outcomes and increases health care costs (11). Short sleep duration is mentioned as a risk factor for weight gain and obesity (12). According to hypotheses regarding the relationship between reduced sleep and obesity, sleep deprivation leads to hormonal changes and hence increases appetite and food intake $(13,14)$. Sleep disorders affect neurohormones resulting in increased caloric intake which may decrease physical activity. Chronic sleep deprivation also causes fatigue and reduced physical activity in individuals. Therefore, sleep duration and quality is associated with obesity (15).

Interrupted sleep in young adults results in metabolic and endocrine changes such as reduced glucose tolerance, 
decreased insulin sensitivity, increased nocturnal cortisol, reduced leptin, increased ghrelin, and increased hunger and appetite (12).

In a study by Tworoger et al to determine the factors associated with sleep in adult women, it was found that body mass index (BMI) was associated with poor quality sleep (16). Also in the study of Beebe et al, symptoms of sleep disorders, delay in sleep onset, and short sleep times were higher in the overweight group compared with the control group (17).

To determine the factors associated with sleep in adult women, Tworoger et al showed that going late to bed, medication, employment, increased day hours, long menstruation cycle, and BMI were associated with low quality sleep (16). But Vgontzas et al demonstrated a relationship between long sleep duration at night and day with weight gain (18).

Since there are limited and contradictory studies about the relationship between sleep quality and overweight and obesity in women of reproductive age and no similar study was found in Iran, the researcher decided to determine the relationship between sleep quality and overweight and obesity in women of reproductive age who were referred to health centers in Yazd.

\section{Materials and Methods}

This descriptive, cross-sectional study with an analytical approach included women of reproductive age (20-35 years) with inclusion criteria who had health records at health centers of Yazd. The women were divided into three groups of normal weight, overweight, and obese.

Inclusion criteria were being Iranian, Muslim, and Yazdi, willing to participate in the study, have at least secondary school education level, women of reproductive age, $\mathrm{BMI} \geq 18.5$, not taking hypnotics, diuretics, and neuropsychiatric medications, lack of conditions that disrupt a person's sleep and frequently waken her according to the person (such as cardiac disease, diabetes, asthma, renal disease, depression, arthritis, and arthralgia), not employed in night shifts, absence of stress and family problems such as severe conflicts, relatives death, accident or serious illness of a family member, financial problems, and losing job and spouse in the past 6 months, absence of pregnancy and lactation, and lack of restless leg syndrome.

Exclusion criteria were unwillingness of the person or her spouse to participate in or continue the study and occurrence of any diseases and consumption of any drugs listed in inclusion criteria during the study.

The sample size was calculated 100 women for each group considering a confidence of $95 \%$, a test power of $80 \%$, and an estimated increase in sleep quality score of $20 \%$ in overweight women, and based on a comparison of two averages (3). But due to the possibility of sample loss, a total of 450 women (150 in each group) was considered as the sample size (design effect $=1.5$ ).

The samples were selected through the cluster random sampling method by selecting a half of Yazd health centers and bases (48 centers) using the computer (http://www. random.org), after which the proper sample size (450 women) was identified according to the demographic information available at each health center and base as a quota for each center or base. The list of records covered by each unit was extracted and the eligible women were divided into normal weight, overweight, and obese groups according to information in the records. They were numbered in the list and then were randomly selected in each group based on quota of each center using a computer.

At this point, a comprehensive information about the reasons, benefits, results, and confidentiality of data, as well as the research method was given to women. If they agreed to participate in the research, they were evaluated in terms of basic information and inclusion criteria based on an inclusion criteria form and if eligible for inclusion, they signed an informed consent form to participate in the study. Their weight and height were measured with a ground scale (seca) and a tape Heightmeter (seca), respectively, which reliability and accuracy were proven by the researcher. Then BMI was calculated and recorded in the personal information form. The data were collected based on the questionnaires completed by the participants.

The questionnaires used in this study were the Pittsburgh Sleep Quality Index (PSQI) including sleep mental quality, delay in sleep onset, sleep duration, good sleep, sleep disorders, hypnotic's consumption, and impaired daily functioning. Scores of each scale were between 0 and 3. Scores $0,1,2$, and 3 in each scale represent normal condition, and weak, moderate, and severe problem, respectively. Finally, the scores of components were summed and a total score was obtained (0 to 21). A total score of higher than 5 means poor sleep quality. This scale has been designed to assess sleep quality during the past month. The second questionnaire consisted of socio-demographic characteristics. The validity of the questionnaires was determined through content and face validity. PSQI is standard and its validity has been already identified (validity 0.86 and reliability 0.89 ) (19). Regarding the validity of the socio-demographic questionnaire, this form was given to faculty members and after gathering their views, the necessary corrections were done.

\section{Statistical Analysis}

The statistical analysis was performed using the SPSS 16. The Ordinal regression was used to determine the relationship between sleep quality scores in women of reproductive age with a BMI score and in the multivariate analysis with adjustment for confounders of ordinal regression was used. A $P$ value $\leq 0.05$ was considered statistically significant.

\section{Results}

In this study, $66 \%$ of normal weight women of reproductive age had a good sleep quality (score $\leq 5$ ), while $77 \%$ of overweight and $93 \%$ of obese women of reproductive age had poor sleep quality (score $>5$ ), respectively (Table 1 ). 
Table 1. Frequency Distribution of Sample Size Based on Total Score of Sleep Quality

\begin{tabular}{|c|c|c|c|c|c|c|}
\hline \multirow{2}{*}{$\begin{array}{l}\text { Total Score of Sleep Quality } \\
\text { Score }(0-21)\end{array}$} & \multicolumn{2}{|c|}{$\begin{array}{c}\text { Women of Childbearing Age With } \\
\text { Normal Weight }\end{array}$} & \multicolumn{2}{|c|}{$\begin{array}{c}\text { Women of Childbearing Age With } \\
\text { Overweight }\end{array}$} & \multicolumn{2}{|c|}{$\begin{array}{c}\text { Women of Childbearing Age With } \\
\text { Obesity }\end{array}$} \\
\hline & No. & $\%$ & No. & $\%$ & No. & $\%$ \\
\hline Score of 5 and Lower than 5 & 99 & 66 & 35 & 23 & 11 & 7 \\
\hline Score of higher than 5 & 51 & 34 & 115 & 77 & 139 & 93 \\
\hline Total & 150 & $100 \%$ & 150 & $100 \%$ & 150 & $100 \%$ \\
\hline
\end{tabular}

The results of this study regarding the sociodemographic characteristics of participants indicated that a direct significant correlation existed between the sociodemographic characteristics (position, economic, social, and lifestyle) and the components of sleep quality, sleep mental quality, delay in sleep onset, sleep duration, and good sleep $(P<0.05)$, but the relationship between the socio-demographic characteristics and the components of sleep disorder, hypnotics consumption, and impaired daily functioning was inversely significant $(P<0.05)$. Finally, the greatest relationship with respect to the calculated beta belonged to the overall quality of sleep $(P=0.001)$. As a result, as the socio-demographic characteristics including position, economic, social, and lifestyle increased, sleep quality improved (Table 2).

The results of this study showed a significant relationship between BMI of normal weight women of reproductive age and sleep quality in general $(P=0.001)$. But there was no significant relationship between BMI and normal weight women of reproductive age and the components of sleep mental quality, delay in sleep onset, sleep duration, and good sleep. However, an inverse significant relationship existed between consumption of hypnotics and impaired daily functioning $(P<0.05)$. The highest correlation according to the amount of calculated beta belonged to the overall quality of sleep. Therefore, as long as women of reproductive age have normal weight, their sleep quality will be better (Table 3 ).

The results of this study showed an inverse relationship between BMI of overweight women of reproductive age and the components of sleep quality, sleep mental quality $(P<0.001)$, while a direct significant relationship existed between weight gain in women of reproductive age and the components of sleep disorder, impaired daily functioning, and consumption of hypnotics $(P=0.001)$. Finally, the highest correlation according to the amount of calculated beta belonged to sleep disorder. As a result, as long as the amount of overweight in women of reproductive age was lower, sleep quality improved and sleep disorder declined (Table 4).

The study also showed an inverse significant relationship between BMI of obesity in women of reproductive age and

Table 2. Multivariate Regression Results for Determining the Relationship Between Personal and Social Characteristics and Sleep Quality Components

\begin{tabular}{|c|c|c|c|c|}
\hline Dependent Variable & Independent Variables & Beta Standard Coefficient & $t$ & $P$ \\
\hline \multirow{7}{*}{$\begin{array}{l}\text { Personal and social } \\
\text { characteristics (job, } \\
\text { financial, social status, } \\
\text { and lifestyle) }\end{array}$} & Sleep quality & 0.779 & 4.337 & 0.001 \\
\hline & Subjective sleep quality & 0.098 & 0.724 & 0.003 \\
\hline & Delay in sleeping & 0.113 & 1.238 & 0.001 \\
\hline & Sleep duration and efficient sleep duration & 0.118 & 1.293 & 0.001 \\
\hline & Sleep disorder & -0.648 & -4.894 & 0.001 \\
\hline & Taking sedating medications & -0.218 & -2.456 & 0.005 \\
\hline & Disorders in daily activities & -0.239 & -4.195 & 0.001 \\
\hline
\end{tabular}

Table 3. Multivariate Regression Results for Determining the Relationship Between Normal Weight in Woman of Childbearing Age and Sleep Quality Components

\begin{tabular}{lllll}
\hline Dependent Variable & Independent Variables & Beta Standard Coefficient & $\boldsymbol{t}$ & $\boldsymbol{P}$ \\
\hline & Sleep quality & 0.621 & 5.654 & 0.001 \\
& Subjective sleep quality & 0.063 & 0.604 & 0.548 \\
& Delay in sleeping & 0.091 & 1.116 & 0.268 \\
Women of childbearing & Sleep duration and efficient sleep duration & 0.099 & 1.401 & 0.165 \\
age with normal weight & Sleep disorder & -0.478 & -5.398 & 0.001 \\
& Taking sedating medications & -0.196 & -2.867 & 0.005 \\
& Disorders in daily activities & -0.292 & -4.226 & 0.001 \\
\hline
\end{tabular}


Table 4. Multivariate Regression Results for Determining the Relationship Between Overweight in Woman of Childbearing Age and Sleep Quality Components

\begin{tabular}{|c|c|c|c|c|}
\hline Dependent Variable & Independent Variables & Beta Standard Coefficient & $t$ & $P$ \\
\hline \multirow{7}{*}{ Overweight } & Sleep quality & -0.387 & -4.788 & 0.001 \\
\hline & Subjective sleep quality & -0.219 & -0.718 & 0.005 \\
\hline & Delay in sleeping & 0.216 & 1.433 & 0.268 \\
\hline & Sleep duration and efficient sleep duration & 0.111 & 1.544 & 0.165 \\
\hline & Sleep disorder & 0.696 & 4.277 & 0.001 \\
\hline & Taking sedating medications & 0.159 & 3.001 & 0.001 \\
\hline & Disorders in daily activities & 0.365 & 5.219 & 0.001 \\
\hline
\end{tabular}

Table 5. Multivariate Regression Results for Determining the Relationship Between Obesity in Woman of Childbearing Age and Sleep Quality Components

\begin{tabular}{|c|c|c|c|c|}
\hline Dependent Variable & Independent Variables & Beta Standard Coefficient & $t$ & $P$ \\
\hline \multirow{7}{*}{$\begin{array}{l}\text { Obesity in women of } \\
\text { childbearing age }\end{array}$} & Sleep quality & -0.529 & -4.283 & 0.001 \\
\hline & Subjective sleep quality & -0.468 & -0.498 & 0.005 \\
\hline & Delay in sleeping & 0.117 & 0.998 & 0.268 \\
\hline & Sleep duration and efficient sleep duration & 0.148 & 1.387 & 0.165 \\
\hline & Sleep disorder & 0.541 & 6.218 & 0.001 \\
\hline & Taking sedating medications & 0.231 & 3.011 & 0.005 \\
\hline & Disorders in daily activities & 0.299 & 3.886 & 0.001 \\
\hline
\end{tabular}

the components of sleep quality and sleep mental quality $(P=0.001)$. While the relationship between obesity in women of reproductive age with components of delay in sleep onset, sleep duration, and good sleep was not significant. In addition, a direct significant relationship existed between BMI of obesity in women of reproductive age and sleep disorder, consumption of hypnotics, and impaired daily functioning $(P<0.05)$. Finally, the highest correlation according to the amount of calculated beta belonged to sleep disorder. Meaning that as long as the rate of obesity was high in women of reproductive age, sleep quality reduced and sleep disorder increased (Table 5).

\section{Discussion}

The results showed that sleep quality was good in the majority of normal weight women of reproductive age, while it was poor in the majority of overweight and obese women of reproductive age, being dramatically worse in obese women.

The results of this study revealed the relationship between BMI of normal weight women of reproductive age and the overall sleep quality. But the relationship between BMI of normal weight women of reproductive age and the components of mental quality, i.e. delay in sleep onset, sleep duration, and good sleep was not significant. The relationship between sleep disorder, consumption of hypnotics, and impaired daily functioning was inverse. Finally, the highest correlation was related to the overall quality of sleep. Therefore, as long as the women of reproductive age had normal weight, the sleep quality was better. It was shown in this study that an inverse relationship existed between BMI of overweight women of reproductive age and sleep mental quality, however, its relationship was directly significant with components of sleep disorder and impaired daily functioning. Finally, the highest relationship belonged to sleep disorder. This means that as long as the amount of overweight was higher in women of reproductive age, sleep quality reduced and sleep disorder increased.

The results of this study also showed an inverse relationship between BMI of obese women of reproductive age and the components of sleep quality and sleep mental quality, but the relationship between obesity in women of reproductive age with the components of delay in sleep onset, sleep duration, and good sleep was not significant. The relationship between BMI of obese women of reproductive age and sleep disorder, consumption of hypnotics, and impaired daily functioning was directly significant. Finally, the highest relationship belonged to sleep disorder. This means that as long as the amount of obesity was higher in women of reproductive age, sleep quality reduced and sleep disorder increased.

Our study showed a direct correlation between sociodemographic characteristics (position, economic, social, and lifestyle) and the components of sleep quality, i.e. sleep mental quality, delay in sleep onset, sleep duration, and good sleep and an inverse significant relationship between the components of sleep disorder, consumption of hypnotics, and impaired daily functioning. Finally, the highest relation belonged to the overall quality of sleep. As a result, as long as the socio-demographic characteristics, i.e. position, economic, social, and lifestyle increased, 
sleep quality improved.

In a study by Rahe et al, $65.3 \%$ and $34.7 \%$ of the participants had good sleep quality (PSQI $\leq 5)$ and poor quality sleep (PSQI $>5$ ), respectively. A significant relationship was also found in this study between low sleep quality and general obesity, body fat mass, and personal, social, and lifestyle factors. This study also showed that poor quality sleep can be a predictor for general obesity and increased body fat mass. In this study, the longest time of sleep, sleep disorder, and sleep disorder during day were assessed (20). The results of this study were consistent with our results in terms of the relationship between poor quality sleep and obesity and the relationship between socio-demographic characteristics and sleep quality.

In a study conducted by Tworoger et al, it was shown that employment, age, and perceived stress are associated with sleep mental quality which is consistent with our study (16).

Thomson et al highlighted that increased quality of sleep as more than 7 hours sleep improved weight loss by as much as $33 \%(21)$, meaning that people with less sleep hours were more likely to become obese and overweight; this result was inconsistent with our study, because there was no relationship in our study between sleep duration, good sleep and overweight, obesity. This inconsistency can be attributed to differences in sample size ( 240 patients in the study of Thompson and 450 people in the present study) and the type of study.

Chaput et al found an inverse relationship between sleep duration and overweight which was inconsistent with the results of our study (22). The study of Ievers-Landis et al also showed that for every one hour of sleep, $41 \%$ will be added to body weight. But among the studied indices, behavioral performance and stress was more associated with sleep duration than obesity; this was inconsistent with our study, which can be attributed to the sociodemographic characteristics of the participants, because this study evaluated adolescents, while the subjects in our study consisted of women of reproductive age. The indices were also different in two studies. Subjects with stressful conditions were not entered in our study. The sample size was also different in two studies (23).

Vorona et al showed that when sleep-wake cycle is objectively measured, severe obesity in human is associated with longer night sleep. The result of this study was also inconsistent with our study. The study was conducted on 1001 patients at 4 levels and had different research tools and methodologies (24). However, Vgontzas et al pointed out a direct correlation between sleep disorder and overweight and obesity, which was consistent with our study (18).

In most reviewed studies, an inverse relationship was found between quality of sleep in general and overweight and obesity, which was consistent with the present study, but regarding the components of sleep quality, it seems that sleep disorder, consumption of hypnotics, and impaired daily functioning were more beneficial in this regard than sleep duration and good sleep. In this regard, changing lifestyle can be very helpful.

\section{Conclusion}

The present study investigated the relationship between sleep quality and overweight and obesity in women of reproductive age. The results of this study revealed that women of reproductive age with overweight and obesity have poor sleep quality. The components of sleep quality, sleep disorders, impaired daily functioning, and consumption of hypnotics have the most important role in reducing sleep quality. According to the relationship between socio-demographic characteristics and sleep quality, it seems that change in lifestyle can improve sleep quality and thus can reduce BMI.

\section{Limitations of the study}

Distribution of questionnaires among respondents in health centers and lack of cooperation of some respondents to answer the questionnaire were among executive problems of this study. Since the present research was carried out only on women of reproductive age referred to the health centers of Yazd, generalization of the results should be performed with more caution.

\section{Ethical Issues}

Written informed consents were obtained from the participants. The ethics committee of the Tabriz University of Medical Sciences approved this study.

\section{Conflict of Interests}

The authors declare no conflict of interests.

\section{Financial Support}

This study was financially supported by Tabriz University of Medical Sciences and the Research Center for Women Health of Tabriz.

\section{Acknowledgments}

The authors would like to thank the Deputy of Education and Research of Tabriz University of Medical Sciences as well as all personnel of Yazd health centers and all participants in the study.

\section{References}

1. Spiegel K, Leproult R, Van Cauter E. Impact of sleep debt on metabolic and endocrine function. Lancet 1999;354(9188):1435-9. doi:10.1016/S01406736(99)01376-8

2. Nunes DM, Mota RMS, de Pontes Neto OL, Pereira EDB, de Bruin VMS, de Bruin PFC. Impaired sleep reduces quality of life in chronic obstructive pulmonary disease. Lung. 2009;187(3):159-63. doi: 10.1007/s00408-009-9147-5.

3. Hung HC, Yang YC, Ou HY, Wu JS, Lu FH, Chang CJ. The association between self-reported sleep quality and overweight in a Chinese population. Obesity. 2013;21(3):486-92. doi:10.1002/oby.20259.

4. Cappuccio FP, D’Elia L, Strazzullo P, Miller MA. Sleep duration and all-cause mortality: a systematic review and meta-analysis of prospective studies. Sleep. 2010;33(5):585- 
92.

5. Knutson KL. Sleep duration and cardiometabolic risk: a review of the epidemiologic evidence. Best Pract Res Clin Endocrinol Metab. 2010;24(5):731-43. doi:10.1016/j. beem.2010.07.001.

6. Chen X, Beydoun MA, Wang Y. Is sleep duration associated with childhood obesity? A systematic review and metaanalysis. Obesity. 2008;16(2):265-74. doi:10.1038/ oby.2007.63.

7. Finucane MM, Stevens GA, Cowan MJ, et al. National, regional, and global trends in body-mass index since 1980: systematic analysis of health examination surveys and epidemiological studies with 960 country-years and 9. 1 million participants. Lancet. 2011;377(9765):557-67. doi:10.1016/S0140-6736(10)62037-5.

8. Flegal KM, Carroll MD, Kit BK, Ogden CL. Prevalence of obesity and trends in the distribution of body mass index among US adults, 1999-2010. JAMA. 2012;307(5):491-7. doi:10.1001/jama.2012.39.

9. Berghöfer A, Pischon T, Reinhold T, Apovian CM, Sharma AM, Willich SN. Obesity prevalence from a European perspective: a systematic review. BMC Public Health. 2008;8(1):200. doi:10.1186/1471-2458-8-200.

10. Moghimi-Dehkordi B, Safaei A, Vahedi M, Pourhoseingholi MA, Pourhoseingholi A, Zali MR. The prevalence of obesity and its associated demographic factors in Tehran, Iran. J Health Develop. 2012;1(1):22-30.

11. Cawley J, Meyerhoefer C. The medical care costs of obesity: an instrumental variables approach. J Health Econ. 2012;31(1):219-30. doi:10.1016/j.jhealeco.2011.10.003.

12. Patel SR, Hu FB. Short sleep duration and weight gain: a systematic review. Obesity. 2008;16(3):643-53. doi:10.1038/ oby.2007.118.

13. Knutson KL, Spiegel K, Penev P, Van Cauter E. The metabolic consequences of sleep deprivation. Sleep Med Rev. 2007;11(3):163-78.

14. Spiegel K, Knutson K, Leproult R, Tasali E, Van Cauter E. Sleep loss: a novel risk factor for insulin resistance and Type 2 diabetes. J Appl Physiol (1985). 2005;99(5):2008-19. doi:10.1152/japplphysiol.00660.2005.
15. Bray GA. Medical consequences of obesity. J Clin Endocrinol Metab. 2004;89(6):2583-9.

16. Tworoger SS, Davis S, Vitiello MV, Lentz MJ, McTiernan A. Factors associated with objective (actigraphic) and subjective sleep quality in young adult women. J Psychosom Res. 2005;59(1):11-9. doi:10.1016/j.jpsychores.2005.03.008.

17. Beebe DW, Lewin D, Zeller M, et al. Sleep in overweight adolescents: shorter sleep, poorer sleep quality, sleepiness, and sleep-disordered breathing. J Pediatr Psychol. 2007;32(1):69-79. doi:10.1093/jpepsy/jsj104.

18. Vgontzas AN, Bixler EO, Chrousos GP, Pejovic S. Obesity and sleep disturbances: meaningful sub-typing of obesity. Arch Physiol Biochem. 2008;114(4):224-36. doi:10.1080/13813450802521507.

19. Taheri S, Lin L, Austin D, Young T, Mignot E. Short sleep duration is associated with reduced leptin, elevated ghrelin, and increased body mass index. PLoS Med. 2004;1(3):e62. doi:10.1371/journal.pmed.0010062.

20. Rahe C, Czira ME, Teismann H, Berger K. Associations between poor sleep quality and different measures of obesity. Sleep Med. 2015;16(10):1225-8. doi:10.1016/j. sleep.2015.05.023.

21. Thomson CA, Morrow KL, Flatt SW, et al. Relationship between sleep quality and quantity and weight loss in women participating in a weight-loss intervention trial. Obesity 2012;20(7):1419-25. doi:10.1038/oby.2012.62.

22. Chaput J, Brunet M, Tremblay A. Relationship between short sleeping hours and childhood overweight/obesity: results from the 'Quebec en Forme'Project. Int J Obes (Lond). 2006;30(7):1080-5. doi:10.1038/sj.ijo.0803291.

23. Ievers-Landis CE, Storfer-Isser A, Rosen C, Johnson NL, Redline S. Relationship of sleep parameters, child psychological functioning, and parenting stress to obesity status among preadolescent children. J Dev Behav Pediatr. 2008;29(4):243-52. doi:10.1097/DBP.0b013e31816d923d.

24. Vorona RD, Winn MP, Babineau TW, Eng BP, Feldman HR, Ware JC. Overweight and obese patients in a primary care population report less sleep than patients with a normal body mass index. Arch Intern Med. 2005;165(1):25-30. doi:10.1001/archinte.165.1.25.

Copyright (C) 2017 The Author (s); This is an open-access article distributed under the terms of the Creative Commons Attribution License (http://creativecommons.org/licenses/by/4.0), which permits unrestricted use, distribution, and reproduction in any medium, provided the original work is properly cited. 